\title{
Investigation of Negative Influences on Ride Comfort Performance of In-Wheel Motor Vehicles with High Unsprung Mass
}

\author{
T.Z. Shi, D.F. Wang, S.M. Chen \\ State Key Laboratory of Automotive Simulation and Control \\ Jilin University \\ Changchun, China, 130022
}

\begin{abstract}
The in-wheel motor electric vehicle is directly driven by motors integrated in wheels. This unique design provides actuation flexibility, energy efficiency and performance potentials. Yet the introducing of in-wheel motor caused massive increment of unsprung mass, which shows negative effect on vehicle ride comfort performance. In this paper, a rigid-elastic coupling multi-body dynamic model of a typical in-wheel motor vehicle is built. Based on the model, vehicle ride comfort performance with different unsprung mass including root mean square of weighted body acceleration, wheel dynamic load, suspension deflection is investigated. The amplitude-frequency characteristics of vehicle is analyzed based on quarter vehicle system.
\end{abstract}

Keywords-in-wheel motor vehicle; ride comfort; unspung mass; rigid-elastic coupling; suspension parameters

\section{INTRODUCTION}

With potentials in emissions and fuel consumption reduction, the electric vehicles including hybrid electric vehicles, plug-in hybrid vehicles and pure electric vehicles are considered as promising vehicle architectures[1-3]. The in-wheel motor electric vehicles (IMEV) which are driven by four independently actuated in-wheel motors are one of those unique designs. With motors directly actuating wheels, the in-wheel motor vehicle is designed without transmission systems or mechanical links[4]. The driving torque of each motor is controlled independently. With the control flexibility and quick response of motors, the vehicle performance can be improved promisingly[5]. The EV and IMEV have achieved impressive driving performance along with the improvements of motors and batteries.

With in-wheel motors integrated in wheels, the unsprung mass of IMEV is increased dramasticly. Studies have shown that with larger unsprung mass, the ride comfort performance of vehicle tends to come down[6,7]. Adjusting the suspension parameters is a effective way to alleviate the negative effect of ride comfort[8]. With different types of in-wheel motor integrated, the unsprung mass are different. In this paper, a rigid-elastic multi-body dynamic model of typical IMEV is built. Based on the model, the influence on vehicle ride comfort performance of different in-wheel motor mass is investigated.

\section{FUll VEHICLE MODELING}

A rigid-elastic coupling muti-body dynamic model of an IMEV is built. The model is composed of double pivot front suspension system; rear suspension system with a flexible body twist beam; steering system; anti-roll bar system; tire system; in-wheel motors; vehicle body and battery cells. Major parameters of the multi-body dynamic model is shown in Table 1.

TABLE I. MAJOR PARAMETERS OF IMEV MODEL.

\begin{tabular}{|l|l|}
\hline Parameter & Value \\
\hline Number of moving parts & 58 \\
\hline Degree of freedom & 74 \\
\hline Curb weight (kg) & 1540 \\
\hline In-wheel motor weight (kg) & 54 \\
\hline Battery cell weight (kg) & 280 \\
\hline Wheel base (mm) & 2389 \\
\hline
\end{tabular}

The mass and geometry parameters were obtained by physical measurements. The bushing, spring and damping characteristics are obtained by disassembling the suspension parts and measuring on hydraulic actuator test bench. The in-wheel motor with motor controller is installed in wheels respectively.

\section{RIDE COMFORT ANALYSIS}

\section{A. Quarter Vehicle Modeling}

The vehicle ride performance can be considered as vibration response of vehicle-road system. In order to analysis the vibration response of vehicle and road interact system, a quarter vehicle system is built. In this model, $\mathrm{m} 1$ and $\mathrm{m} 2$ are the unsprung and sprung mass of vehicle respectively; Z1 and Z2 are vertical displacement of wheel and body; $\mathrm{K}$ is the suspension spring stiffness; Kt is the tire vertical stiffness; $\mathrm{C}$ is damping coefficient of damper system; q represents for the irregularity of road. The dynamic equation of the quarter vehicle model is:

$$
\left\{\begin{array}{l}
m_{2} \ddot{z}_{2}+C\left(\dot{z}_{2}-\dot{z}_{1}\right)+K\left(z_{2}-z_{1}\right)=0 \\
m_{1} \ddot{z}_{1}+C\left(\dot{z}_{1}-\dot{z}_{2}\right)+K\left(z_{1}-z_{2}\right)+K_{t}\left(z_{1}-q\right)=0
\end{array}\right.
$$

Solve Eq. (1) to obtain the transfer function of body and wheel displacement in response of q:

$$
H(j \omega)_{z_{1}-q}=\frac{K_{t}\left(-\omega^{2} m_{2}+j \omega C+K\right)}{U}
$$




$$
\begin{gathered}
H(j \omega)_{z_{2}-q}=\frac{K_{t}(j \omega C+K)}{U} \\
U=\left(-\omega^{2} m_{2}+j \omega C+K\right)\left(-\omega^{2} m_{1}+j \omega C+K+K_{t}\right)-(j \omega C+K)^{2}(4)
\end{gathered}
$$

\section{B. Body Acceleration Response}

The body acceleration response is often considered as an evaluation index for ride comfort performance. The amplitude-frequency characteristics $|H(j \omega)|_{i-\dot{q}}$ of body acceleration in response of road irregularity is:

$$
\begin{gathered}
|H(j \omega)|_{i-\dot{q}}=2 \pi f \frac{K_{t}}{K}\left[\frac{1+4 \xi^{2}\left(f / f_{0}\right)^{2}}{V}\right]^{\frac{1}{2}} \\
V=\left[1-\left(f / f_{0}\right)^{2}\left(1+\frac{K_{t}}{K}-\frac{m_{1}}{m_{2}}\left(f / f_{0}\right)^{2}\right)-1\right]^{2}+4 \xi^{2}\left(f / f_{0}\right)^{2}\left[\frac{K_{t}}{K}-\left(\frac{m_{1}}{m_{2}}+1\right)\left(f / f_{0}\right)^{2}\right]^{2}
\end{gathered}
$$

where $f_{0}=\sqrt{k / m_{2}} / 2 \pi$ defines the natural frequency of vehicle body, $\xi=C / 2 \sqrt{K \times m_{2}}$ is the damping ratio of quarter vehicle system. The root mean square of body acceleration is given as:

$$
\sigma_{\ddot{z}}=\sqrt{4 \pi^{2} G_{q}\left(n_{0}\right) n_{0}^{2} v \int_{0}^{\infty}|H(j \omega)|_{\ddot{z}-\dot{q}}^{2} d f}
$$

Eq. (2), (3) and (7) show the vibration characteristics of quarter vehicle system. It can be seen that the factor $\mathrm{m} 1 / \mathrm{m} 2$ is a key factor influences acceleration response. Figure 1 shows the relationship between body acceleration and mass ratios in different speeds. The result is obtained by adjust the motor mass of multi-body full vehicle model on B class pavement. With larger $\mathrm{m} 2$ value, the body acceleration tends to increasing slightly.

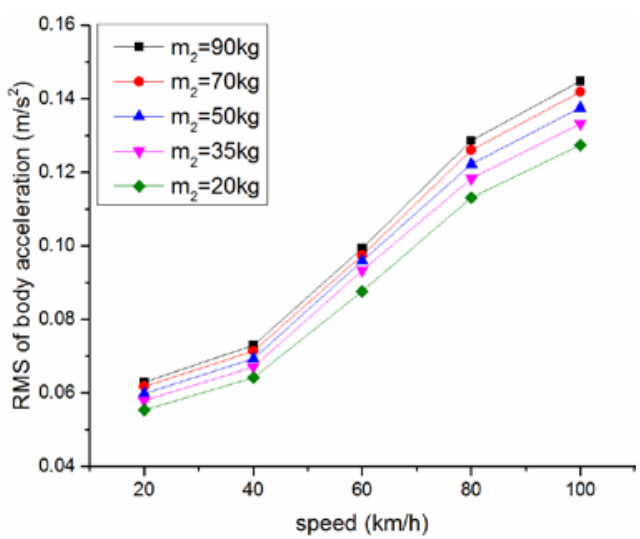

FIGURE I. BODY ACCELERATION RESPONSE.

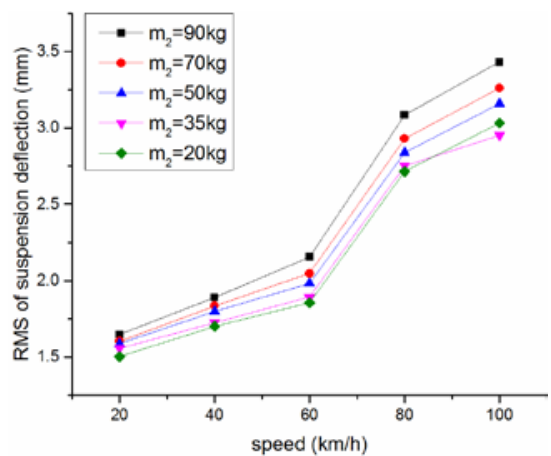

FIGURE II. SUSPENSION DEFLECTION RESPONSE.

\section{Suspension Deflection Response}

The root mean square of suspension deflection $\sigma \mathrm{fd}$ is described as:

$$
\left\{\begin{array}{l}
\sigma_{f d}=\sqrt{4 \pi^{2} G_{q}\left(n_{0}\right) n_{0}^{2} v \int_{0}^{\infty}|H(j \omega)|_{z-\dot{q}}^{2} d f} \\
|H(j \omega)|_{z-\dot{q}}=\frac{f \square K_{t} / K}{2 \pi f_{0}^{2} \sqrt{B}}
\end{array}\right.
$$

Adjust the motor mass of multi-body full vehicle model and run the models on B class road, relationship between suspension deflection and mass ratios is obtained as in Figure 2. With higher speed, the influence of higher unsprung mass is greater. The increment is $11.65 \%$ at maximum.

\section{Wheel Dynamic Load Response}

The amplitude-frequency characteristic of wheel dynamic load $\sigma \mathrm{Fd}$ in response of speed and the root mean square of wheel dynamic load is given as:

$$
\left\{\begin{array}{l}
|H(j \omega)|_{F_{d}-\dot{q}}=2 \pi f \frac{K_{t}}{K} \sqrt{\frac{\left[\left(f / f_{0}\right)^{2}-\left(1-m_{1} / m_{2}\right)\right]^{2}+4 \xi^{2}\left(f / f_{0}\right)^{2}}{\left(1-m_{1} / m_{2}\right) V}} \\
\sigma_{F_{d}}=\sqrt{4 \pi^{2} G_{q}\left(n_{0}\right) n_{0}^{2} v \int_{0}^{\infty}|H(j \omega)|_{F_{d}-\dot{q}}^{2} d f}
\end{array}\right.
$$

Figure 3 shows the relationship between wheel dynamic load and mass ratios on B class pavement of different speed. With higher speed and higher unsprung mass, the wheel dynamic load is higher. The greatest increment is $40.4 \%$, which means this index is heavily influenced.

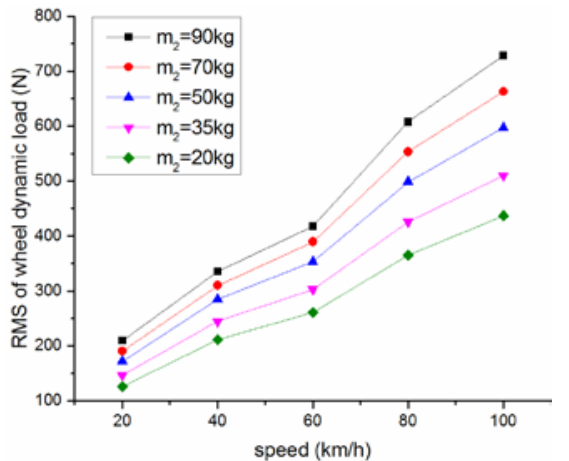

FIGURE III. WHEEL DYNAMIC LOAD RESPONSE. 


\section{CONCLUSIONS}

A rigid-elastic coupling multi-body dynamic model of a in-wheel motor vehicle was built. The ride comfort performance of different mass ratios is investigated. The vibration characteristics of vehicle system is analyzed based on the quarter vehicle model.

Negative influence on vehicle ride comfort is investigated. The body acceleration is influenced by the change of unsprung mass. The largest increment on suspension deflection is $11.65 \%$. The wheel dynamic load is influenced heavily in a increment of $40.4 \%$. For ride comfort performance of vehicle with high unsprung mass, a higher damper coefficient is suggested.

\section{ACKNOWLEDGMENT}

This research work was supported by National Natural Science Foundation project of China (No. 51205152). The authors would like to express their appreciations for the above fund support.

\section{REFERENCE}

[1] R. Wang, Y. Chen and D. Feng et al., Development and performance characterization of an electric ground vehicle with independently actuated in-wheel motors , J Power Sources 196 (2011) 3962-3971.

[2] K. M. Rahman, K. M. Rahman and N. R. Patel et al., Application of Direct-Drive Wheel Motor for Fuel Cell Electric and Hybrid Electric Vehicle Propulsion System, Ieee T Ind Appl 42 (2006) 1185-1192.

[3] H. Alipour, M. Sabahi and M. B. B. Sharifian, Lateral stabilization of a four wheel independent drive electric vehicle on slippery roads, Mechatronics (2014).

[4] L. Rambaldi, E. Bocci and F. Orecchini, Preliminary experimental evaluation of a four wheel motors, batteries plus ultracapacitors and series hybrid powertrain , Appl Energ 88 (2011) 442-448.

[5] W. Kim, K. Yi and J. Lee, Drive control algorithm for an independent 8 in-wheel motor drive vehicle, Journal of Mechanical Science and Technology 25 (2011) 1573-1581.

[6] G. Nagaya, Y. Wakao and A. Abe, Development of an in-wheel drive with advanced dynamic-damper mechanism , Jsae Rev 24 (2003) 477-481.

[7] D. Hrovat, Influence of unsprung weight on vehicle ride quality , J Sound Vib 124 (1988) 497-516.

[8] S. Chen and D. Wang, Optimization of Vehicle Ride Comfort and Controllability Using Grey Relational, Journal of Grey System 23 (2011) 369-380. 